\title{
Chromium nanostructures formed by dewetting of heteroepitaxial films on $W(100)$
}

Article

Published Version

Bennett, R. A., Mulley, J. S., Etman, H. A., Sparkes, A., Eralp, T., Held, G., Cavill, S. A. and Dhesi, S. S. (2012) Chromium nanostructures formed by dewetting of heteroepitaxial films on W(100). Physical Review B, 86 (4). 045454. ISSN 1098-0121 doi: https://doi.org/10.1103/PhysRevB.86.045454 Available at http://centaur.reading.ac.uk/28915/

It is advisable to refer to the publisher's version if you intend to cite from the work. See Guidance on citing.

To link to this article DOI: http://dx.doi.org/10.1103/PhysRevB.86.045454

Publisher: American Physical Society

All outputs in CentAUR are protected by Intellectual Property Rights law, including copyright law. Copyright and IPR is retained by the creators or other copyright holders. Terms and conditions for use of this material are defined in the End User Agreement. 


\section{CentAUR}

Central Archive at the University of Reading

Reading's research outputs online 


\title{
Chromium nanostructures formed by dewetting of heteroepitaxial films on W(100)
}

\author{
Roger A. Bennett, ${ }^{1, *}$ James S. Mulley, ${ }^{2}$ Haitham A. Etman, ${ }^{1}$ Ailsa Sparkes, ${ }^{2}$ Tugce Eralp, ${ }^{1}$ Georg Held, ${ }^{1}$ \\ Stuart A. Cavill, ${ }^{3}$ and Sarnjeet S. Dhesi ${ }^{3}$ \\ ${ }^{1}$ Department of Chemistry, University of Reading, Reading, Berkshire, RG6 6AF, United Kingdom \\ ${ }^{2}$ Department of Physics, University of Reading, Reading, Berkshire, RG6 6AF, United Kingdom \\ ${ }^{3}$ Diamond Light Source Ltd, Harwell Science and Innovation Campus, Didcot, Oxfordshire, OXI1 ODE, United Kingdom
}

(Received 8 May 2012; published 30 July 2012)

\begin{abstract}
In this paper, we report the surprising formation of square-based facetted islands with linear dimension of the order of $500 \mathrm{~nm}$ upon dewetting of a Cr multilayer on W(100). We show that these square islands are composed of inclined facets surrounding a depressed center such that the facet slopes inward with the outer edges of the islands thicker than the centers. The islands' shapes do not represent traditional equilibrium crystal shapes as expected for a Wulf construction. In situ UV and x-ray photoelectron emission microscopy allied to spatially resolved spectroscopy throws considerable light on the nature of the dewetting and shows that the metal surface between the islands remains covered by a thin pseudomorphic wetting layer of $\sim 1 \mathrm{ML}$. Low-energy electron diffraction and scanning tunneling and atomic force microscopies allow quantification of facet slopes, and we identify a predominance of tilted $\operatorname{Cr}(100)$ facets $\pm 5^{\circ}$ off of the substrate normal bound by (210) planes at $\sim 26^{\circ}$. The epitaxial $\mathrm{Cr}$ islands adopt the bulk $\mathrm{Cr}$ lattice constant but are tilted with respect to the surface normal. We suggest that the $\mathrm{Cr}$ crystallite tilting creates a vicinal-like interface structure that determines the island morphology.
\end{abstract}

DOI: 10.1103/PhysRevB.86.045454

PACS number(s): 68.55.-a, 68.37.-d, 68.35.bd, 68.65.-k

\section{INTRODUCTION}

Epitaxial growth on refractory metal surfaces, and especially of magnetic materials, has a long and distinguished history and has directly led to the understanding required to make magnetoresistive and spin-polarized electronic devices and components which form the basis of much IT infrastructure. $\mathrm{Cr}$ on $\mathrm{W}$ is a leading candidate for the fabrication of antiferromagnetic scanning tunneling microscopy tips ${ }^{1-3}$ despite being predicted to have a ferromagnetic ground state at 1 monolayer (ML) and being thermodynamically unstable at higher coverage. ${ }^{4}$ The structure of the tip apex is key to its spin sensitivity and in a monocrystalline and epitaxial $\mathrm{Cr}$ on $\mathrm{W}$ tip was shown to form nanoclusters acting as nanotips. ${ }^{2}$ In growing thin films, it is possible to induce stress, strain, and imprint structural motifs by epitaxy, which further critically influence magnetic behavior. In some systems, alloying can also occur, as is seen for $\mathrm{Cr}$ on $\operatorname{Pt}(111) ;^{5}$ however, for tungsten no intermixing is observed. $\mathrm{Cr}$ adsorption on the W(100) surface has received relatively little attention ${ }^{6}$ until recently when Guo et al. employed the epitaxial growth of $\mathrm{Cr}$ on $\mathrm{W}(100)$ and post oxidation to form chromium oxides controllably. ${ }^{7}$ In a later low-energy electron microscopy (LEEM) study, ${ }^{8}$ the growth of multilayers of Cr on W(100) was investigated and particularly the dewetting at $\sim 3$ ML coverage of films grown above $575 \mathrm{~K}$. Despite the same crystal structure [body centered cubic (bcc)], the large lattice mismatch between $\mathrm{Cr}$ (lattice constant $=2.88 \AA$ ) and W $(3.16 \AA)$ drives structural changes in the films due to strain accumulation with thickness. At temperatures below $575 \mathrm{~K}$, the films grew at least one, and often two, pseudomorphic overlayers (depending upon temperature) with $\mathrm{Cr}$ adopting the $\mathrm{W}$ crystal structure and lattice constants before roughening. Above $575 \mathrm{~K}$, the third monolayer spontaneously dewets to form three-dimensional islands by removal of $1 \mathrm{ML}$ from the pseudomorphic layers on the substrate.
In contrast, Fe on W(100), which is also bcc with an almost identical lattice constant of $2.87 \AA$ to $\mathrm{Cr}$, has been more intensively studied. Recently, this system has been shown ${ }^{9}$ to form dislocation networks in multilayers grown under similar conditions to the $\mathrm{Cr}$ work. These networks propagate in the film such that islands become strain relaxed in one in-plane direction, but fully strained in the other. Interestingly, these islands grow and accrete adatoms at the strained island edges so that the islands elongate in the same direction as the dislocations. These island shapes are dictated by growth kinetics rather than thermodynamic equilibrium. At $500 \mathrm{~K}$ and above $4 \mathrm{ML}$, the growth mode changes subtly again as dislocated islands are so much more stable than the strained wetting layer. The islands deplete the substrate of several monolayers of $\mathrm{Fe}$ and again form long islands, although in this case the dislocation bundles run orthogonal to the long axis of the islands and the habit is closer to that expected of thermodynamic equilibrium. The authors suggest that these long self-assembled islands may be magnetically useful as interconnects in future spin electronics.

$\mathrm{Cr}$ on W(110) also forms elongated striped islands aligned along the $\langle 001\rangle$ direction when grown at elevated temperature and dewet. ${ }^{10}$ The first two monolayers grown are less stable than the fourth, and subsequent layers therefore dewet to thicken the fourth layer. The exceptional anisotropy in growth shape was suggested to be driven by dislocation and strain relaxation or by high stability of $\mathrm{Cr}(001)$-oriented step edges. The substrate step-edge density and orientation was responsible for the initiation of dewetting as the films can thicken by overgrowing a descending train of substrate step edges, a mechanism identified for $\mathrm{Fe}$ and $\mathrm{Cr}$ growth on $\mathrm{Mo}(110){ }^{11,12}$

There has been intense interest in developing strong physical models and theories for strained island shapes grown by vapor deposition on well-defined substrates. Initial work by Tersoff and Tromp ${ }^{13}$ introduced a theory for critical island sizes at which shape transitions occur from square compact, 
trapezoidal cross sectioned, to elongated and strained islands with characteristic narrow widths. As these islands grow, the cross section moves from trapezoidal to triangular to minimize the total energy. This theory has been highly successful in describing growth in semiconductor systems where quantum dots and wires self-assemble and also in transition-metal oxide strained epitaxy. ${ }^{14}$ Recent theoretical developments have focused on the role of vicinal surfaces in directing island shapes of which two are very relevant here. The first showed that highly asymmetric island shapes can develop during epitaxial growth in nonzero miscut systems and that in these systems there is no barrier to island formation in a facetted system in contrast to the case of high-symmetry substrates. ${ }^{15}$ The second explores the elongation and drift of nanowedge islands on vicinal substrates in both early stages, where a film dewets and islands grow, and the later stage after growth. ${ }^{16}$ This dewetting and island advancement in the direction of descending substrate steps have been shown to be a viable method of fabricating islands' arrays by directed self-assembly. ${ }^{17}$ Very recently, the theory behind the dewetting of crystalline films has identified that the creation of facetted rims around dewet holes controls the kinetics of hole growth. Exponents of $\sim t^{1 / 4}$ and $\sim t^{2 / 5}$ are found for the rate of propagation of the hole rim for continuum models, yet the presence of facets changes this to $\sim t^{1 / 2}$ due to the requirement of a nucleation event on the facet.

In this paper, we describe the growth of chromium islands which do not conform to the expected shape of strained islands. These islands can be formed both by dewetting of a metastable multilayer film or by nucleation and growth on a wetting layer. The morphologies are determined by various electron and scanning probe microscopies and represent a new class of shapes ${ }^{18}$ and a challenge to theory. The new morphologies are square-based facetted ring structures with well-defined facet planes inclined with respect to the substrate.

\section{EXPERIMENTAL METHODS}

Cr films were grown in three ultrahigh vacuum (UHV) systems and on two W(100)-oriented single crystals with varying average step densities leading to mean terrace widths from $\sim 70 \AA$ on crystal $\mathrm{A}$ to $240 \AA$ on crystal B. In the latter, the steps were observed to bunch, so a wide range of sizes were apparent. The results on both crystals were almost identical and where differences arise they will be highlighted in the text. The first chamber, based in Reading, has been described previously. ${ }^{19,20}$ The ultrahigh-temperature sample mount initially employed a W5\%Re/W26\%Re thermocouple spot welded to the edge of the W(100) crystal, which was later removed (after calibrating the heating power to temperature) to enable scanning tunneling microscopy (STM) experiments. The STM is homemade ${ }^{21}$ and controlled by an Oxford Instruments TOPS3 system calibrated on O-c $(6 \times 2)$ on $\mathrm{Cu}(110)$ (Ref. 22) and $\operatorname{Re}(0001) .{ }^{23}$ Stated tunneling biases are applied to the sample with the tip at virtual ground. The second apparatus is installed as the end station on beamline I06 of the Diamond synchrotron. It is composed of a low-energy emission microscopy/photoelectron emission microscopy (LEEM/PEEM) chamber and a sample preparation chamber with Omicron RV low-energy electron diffraction (LEED), sample heating manipulator, and gas dosing facilities. The sample mount includes a W5\%Re/W26\% Re thermocouple mounted close to the crystal. Some experiments were also performed on beamline 4.2 of the Daresbury Synchrotron Radiation Source, although only the LEED patterns recorded will be considered here. The same Oxford Applied Research EGN4 $e$-beam evaporator was mounted on the various chambers and used to deposit the Cr films in all experiments.

The W(100) crystals were cleaned by identical standard techniques of $1 \times 10^{-7}$ mbar oxygen roasting at $1500 \mathrm{~K}$ with two subsequent flashes to $2300 \mathrm{~K}$ to desorb residual oxygen. Once cool to below $\sim 370 \mathrm{~K}$, the LEED pattern showed sharp integral order spots and a faint $\mathrm{c}(2 \times 2)$ fuzzy background due to the residual clean surface $(\sqrt{2} \times \sqrt{2}) R 45^{\circ}$ reconstruction present above the transition temperature of $\sim 230 \mathrm{~K}^{24}$ In this condition, the ultraviolet photoemission spectroscopy showed clear surface state peaks at $\sim 0.4,1.0,1.8$, and $4.5 \mathrm{eV}$; both the LEED and UPS results are reported to only occur if the surface is extremely clean. $\mathrm{Cr}$ was deposited at normal incidence in the laboratory-based experiments at a flux of $1 \mathrm{ML} / 150 \mathrm{~s}$ evaluated by a breakpoint in X-ray photoemission spectroscopy (XPS) intensities versus time. The same flux, but at an incidence angle of $\sim 45^{\circ}$, was used in the synchrotron experiments.

All synchrotron-based measurements presented in this article were taken with the spectroscopic photoemission and lowenergy electron microscope (SPELEEM) on beamline I06. The beamline is designed to allow high spatial resolution imaging of surfaces using x-ray absorption spectroscopy (XAS) or XPS as the contrast mechanism. The SPELEEM produces direct images of the surface after collecting and magnifying the photoelectrons emitted from the sample allowing $\mathrm{X}$-ray photoemission electron microscopy (XPEEM) to be performed with a spatial resolution of $50 \mathrm{~nm}$ and an energy resolution of less than $300 \mathrm{meV}$. For further details of the beamline, see Ref. 25. At the time the experiments were undertaken, the monochromator was calibrated at the $N K$ edge. Away from this energy, the photon energy systematically deviates from true calibration due to the grating angle error, and the spectra in this paper are uncorrected for this effect.

For direct comparison between the experimental systems, a film was grown and measured in the laboratory, transferred in air to ex situ imaging by contact mode atomic force microscopy (Topometrix Explorer AFM, images prepared in WSXM software ${ }^{26}$ ) and field emission secondary electron microscopy (FEI ESEM in high vacuum mode) at the Centre for Advanced Microscopy at Reading. It was then mounted at the synchrotron and outgassed at $573 \mathrm{~K}$ and imaged in the XPEEM for comparison. Further films were grown in the XPEEM system.

\section{RESULTS}

\section{A. Film growth: Low-energy electron diffraction}

Identical LEED patterns were observed in all experimental chambers for clean and chromium-covered surfaces. For the growth of the thinner films, our LEED analysis compares favorably with that of Man et al., ${ }^{8}$ which also supports our coverage assignment from XPS breakpoints (not shown). We did not, however, identify their $(4 \times 4)$ structure for 


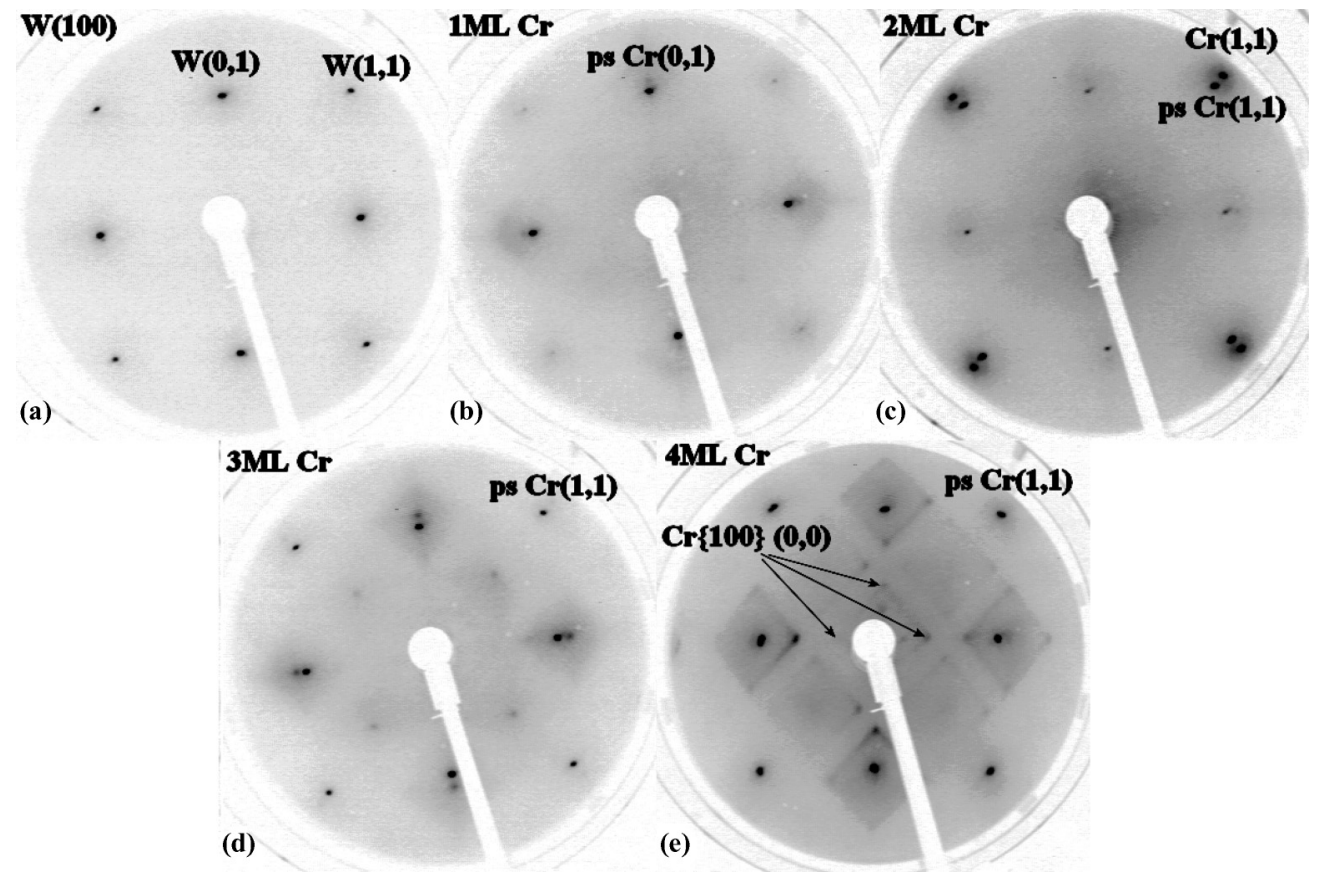

FIG. 1. LEED images acquired at $97-\mathrm{eV}$ beam energy for a range of $\mathrm{Cr}$ coverages with $\mathrm{Cr}$, W, and pseudomorphic $\mathrm{Cr}(\mathrm{ps} \mathrm{Cr})(0,1)$ and $(1,1)$ indexed spots indicated. (a) From the clean W(100) surface; (b) from 1-ML Cr and shows no additional LEED spots indicating a pseudomorphic (ps) layer has grown; (c) from 2-ML Cr showing diffraction spots at both W(100) (from ps Cr) and bulk lattice Cr(100) positions with (1,1) spots highlighted indicating common normal vectors of the exposed (100) surfaces; (d) a typical 3-ML LEED pattern displaying square structured background to bulklike $\mathrm{Cr}$ spots and to a lesser extent on the ps $\mathrm{Cr}$; (e) shows the diffraction pattern from a 4-ML film (and similar for thicker films) in which the square background has developed centered on the positions at which the bulk Cr spots were previously apparent. New bulklike $\mathrm{Cr}(0,0)$ spots appear at fixed angle from the substrate $(0,0)$ and do not move with beam energy, indicating tilted $\mathrm{Cr}\{100\}$ facets.

films of $>3 \mathrm{ML}$ grown in a narrow temperature regime (575-630 K). Detailed discussion of the LEED intensity variation (LEED-IV) results for thin Cr films in the 1-5 ML range will be presented elsewhere ${ }^{27}$ here, we concentrate on the LEED structure of thinner films and their relevance to the development of nanostructured films. Figure 1 shows representative LEED data taken of the clean W(100) surface [Fig. 1(a)] and several different Cr film thicknesses all grown at $673 \mathrm{~K}$ and annealed for $5 \mathrm{~min} / \mathrm{ML}$. For $1 \mathrm{ML}$ [Fig. 1(b)], the growth is pseudomorphic and the LEED displays a sharp $(1 \times 1)$ pattern. For 2 ML [Fig. 1(c)], the films show additional diffraction features due to bulk lattice constant $\mathrm{Cr}$ integral order spots that fall just outside the $\mathrm{W}$ (or pseudomorphic $\mathrm{Cr}$ ) spots. The positions agree well with the expected $\sim 9 \%$ lattice mismatch $(\mathrm{W}=3.16 \AA, \mathrm{Cr}=2.88 \AA)$. At this stage, both $\mathrm{Cr}(100)$ and $\mathrm{W}(100)$ normals are colinear. For films of

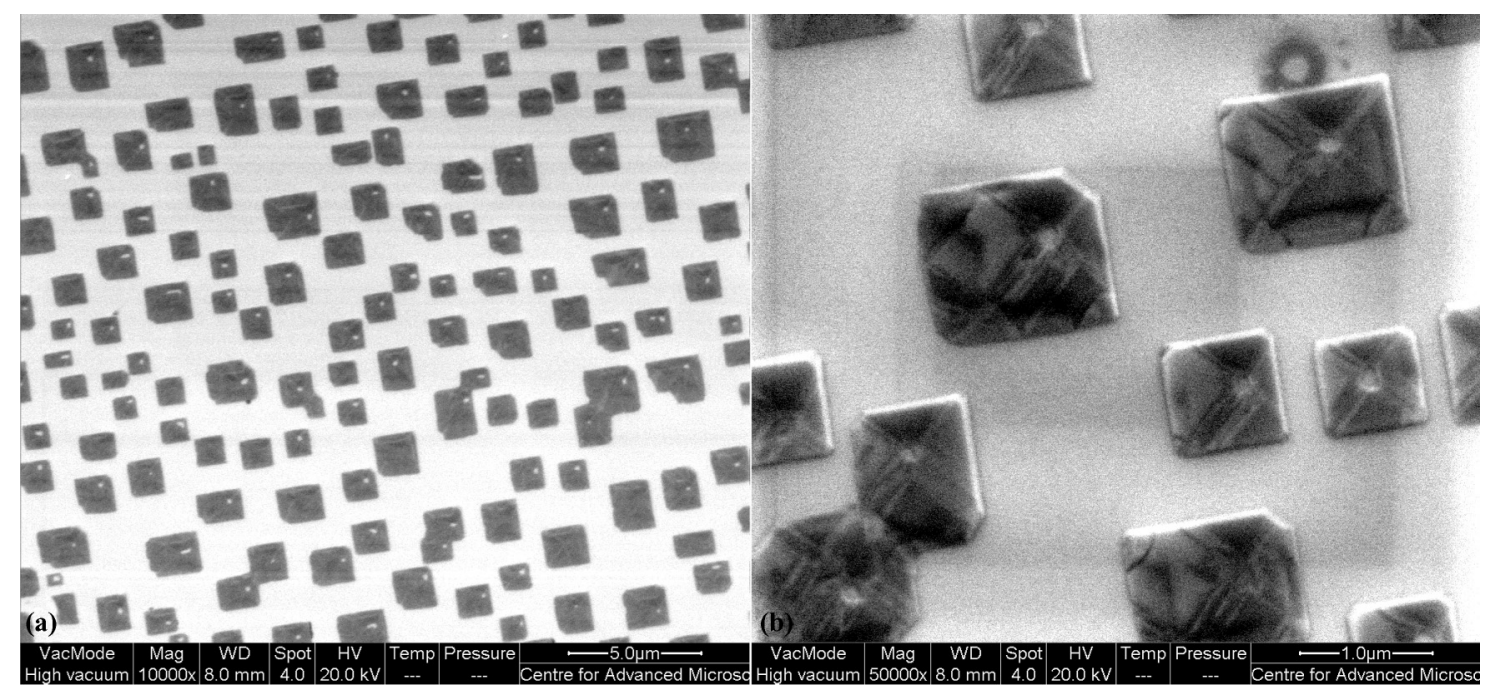

FIG. 2. FE-SEM images acquired at $20 \mathrm{keV}$ in secondary electron mode of structures grown in UHV and transported in air for imaging. (a) Shows a large area image of the island array, the scale bar is $5 \mu \mathrm{m}$; (b) a higher-resolution image of the square islands showing a clear faceted structure, the scale bar is $1 \mu \mathrm{m}$. 
3 ML [Fig. 1(d)], the bulklike Cr spots develop a square halo of increased intensity, which is centered on the position of the $\mathrm{Cr}$ spot and aligned with the $\mathrm{W}$ lattice. A faint $\mathrm{c}(2 \times$ 2) pattern is also present with lattice constant of $\mathrm{Cr}$ rather than W. For thicker films (4-20 ML investigated here), the square halo develops into clear spots in each corner with a drop in the background intensity [Fig. 1(d)] at $4 \mathrm{ML}$, for example. Diffraction features in the W(100) positions reappear and the new features on the square background can all be indexed to the Cr lattice constant in a family of $\{100\}$ planes.

Analysis at variable beam energy shows that these superstructure spots in thicker films do not converge towards the $\mathrm{W}(100)$ surface normal. They instead arise from $\{100\}$ facets inclined $\sim 5^{\circ}$ from the substrate normal with a lattice constant consistent with $\mathrm{Cr}$. The specular spots for each of these four facets are in the square formation closest to the electron gun. A faint $\mathrm{c}(2 \times 2)$ pattern also remains, which is associated with each of the four tilted $\mathrm{Cr}(100)$ domains, producing additional subtle square patterns.

\section{B. Ex situ microscopy}

A thick film was grown and annealed to form a strongly facetted LEED pattern and then removed from the UHV chamber to air and imaged by FE-SEM at $20 \mathrm{kV}$. The images were taken using the secondary electron detector, which is sensitive to surface structure. Figure 2 shows representative images of the island arrays formed on the surface of the crystal at various magnifications. Square islands are readily apparent across the crystal surface [Fig. 2(a)], with lateral dimensions of $\sim 1 \mu \mathrm{m}$. Figure 2(b) shows a higher-resolution view of typical islands in which it is clear that they are facetted and have fine structure. They also notably have a bright central spot to which we shall return. Towards the edge of the crystal, partial shadowing from the $\mathrm{Cr}$ deposition (a shield with aperture is installed between evaporator and sample to prevent covering the LEED screen, which is in line of sight of the evaporator) leads to a different morphology. In this region, the islands are more heterogeneous, more widely spaced, and have an " $L$ " shape (see SupplementalMaterial ${ }^{28}$ ). These islands appear as two rectangular sections joined at right angles and it is commonly seen that each arm has a bright stripe down the

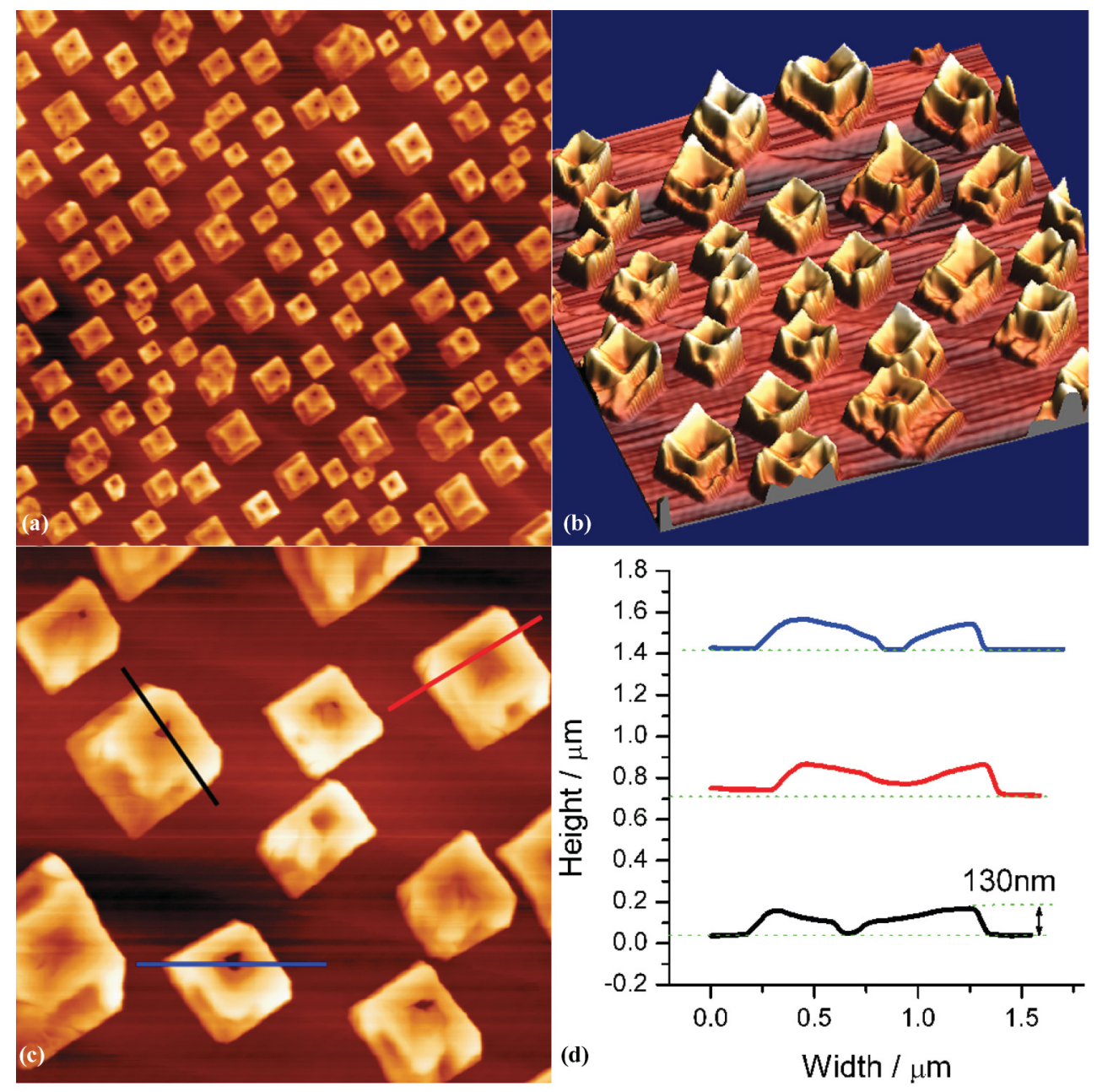

FIG. 3. (Color online) Contact mode AFM images taken ex situ of the Cr on W(100) films. (a) 20- $\mu$ m square image showing uniformly shaped square-based islands with central depressions. (b) 3D rendered $10-\mu \mathrm{m}$ image in which the central pit and facets are highlighted. (c) 5- $\mu \mathrm{m}$ image of the islands at higher magnification with line profiles marked. (d) Line profiles taken from image (c) in both fast scan direction (horizontal) and orthogonal to facets (diagonal). 
center or a bright dot at the apex. Rather interestingly, the islands all "point" in the same direction and there is some indication that their formation is dependent upon the step edges of the substrate. The arms and apex appear to be similarly structured to the square islands.

Islands can also nucleate upon substrate features such as step bunches or possibly (110) microfacets that run across some of our images diagonally. In this region, the islands are sparser on the terrace close to the line defect and adopt a dense square habit on the defect. On very wide field-of-view images, it is possible to discern meandering rows of islands with locally different island number density. Some selected images are included as Supplemental Material. ${ }^{28}$ Elemental mapping showed marginally that the islands were $\mathrm{Cr}$ rich with respect to the terrace.

While scanning electron microscope (SEM) images proved informative with regard to the morphology of the film, they do not provide full three-dimensional (3D) reconstructions of the island shape and hence we turned to contact AFM to map out the topographic details of the structures. Figure 3 displays the AFM images which reveal the truly novel habit these islands adopt. Figure 3(a) shows a representative group of islands from the unshadowed region of the crystal which show the facetted nature of the crystals such that the upturned facets face inward towards the center of the crystal and that the bright central feature seen in the SEM images is actually a pit. A three-dimensionally rendered image [Fig. 3(b)] and line profiles [Figs. 3(c) and 3(d)] allow us to visualize and quantify the island shapes. The exterior steeper sides of the islands are inclined by about $\sim 23^{\circ}$ from horizontal, which is shallower than the sidewalls of the contact mode tip (the manufacturers state $\left.35^{\circ}\right),{ }^{29}$ and hence are a true representation of the morphology and not an artifact arising from tip morphology. The pit in the center of the islands is also steep and can extend down to the terrace on which the islands reside. The four inward inclined facets present fairly well-defined angles with respect to the substrate of between $3^{\circ}$ and $8^{\circ}$. The overall shape is reminiscent of a square-based castle with battlements and an inner courtyard. The overall height of the islands is rather low at just $\sim 120 \mathrm{~nm}$ and so the majority of the island facet forms a low wedge shape. The AFM images tend to exaggerate the $z$ dimension so we have displayed the line profiles with identical

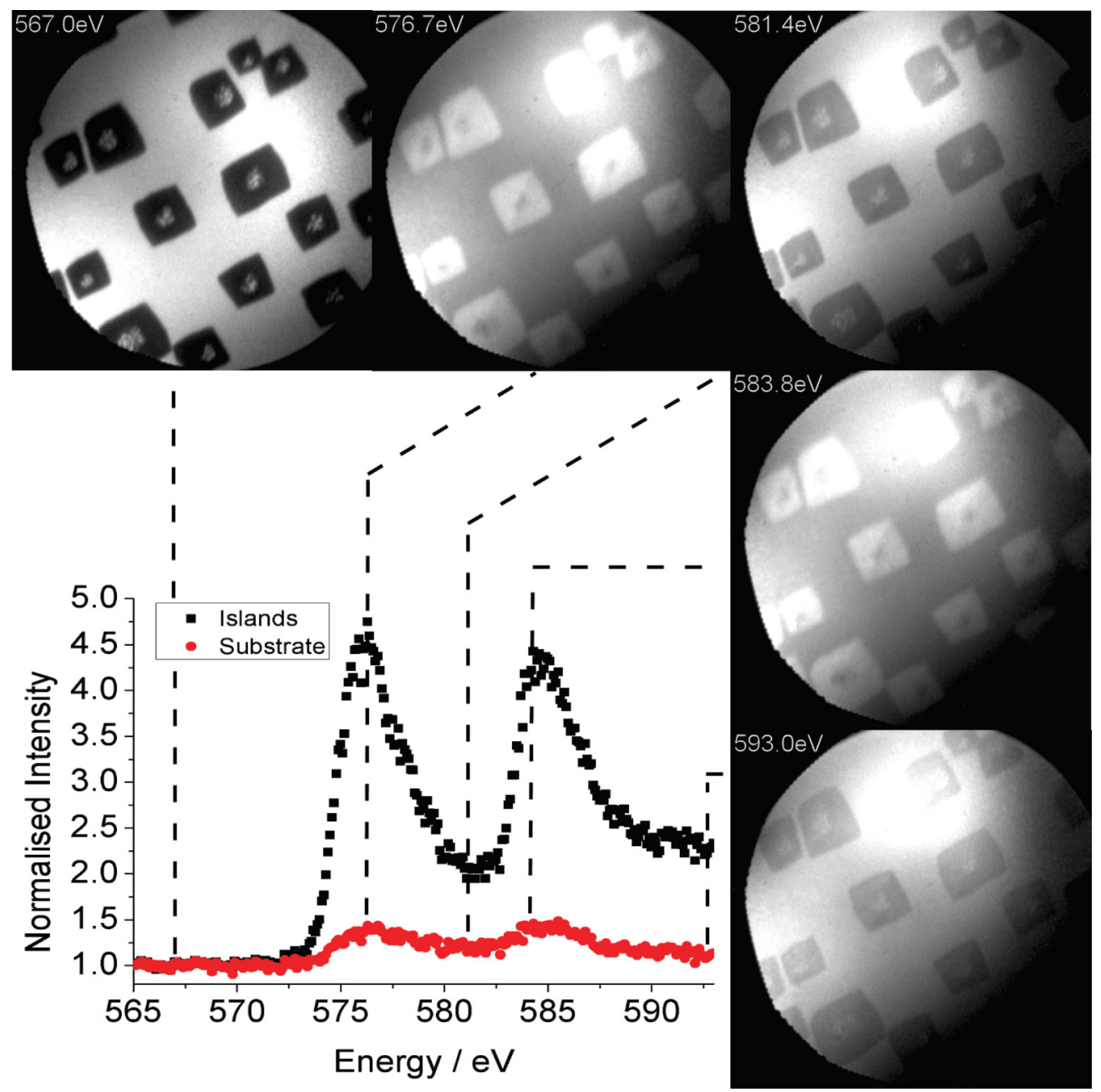

FIG. 4. (Color online) $\mathrm{Cr} L$-edge XAS spectra and associated images (15- $\mu \mathrm{m}$ FOV) taken during a typical XAS experiment on a notionally 12-ML-thick film deposited and annealed in UHV at $803 \mathrm{~K}$ for $15 \mathrm{~min}$. The contrast pre-edge is due to islands attenuating the substrate photoelectrons and secondary electrons, while on the edge the islands photoemit stronger than the thin layer wetting the substrate. The islands show internal fine structure in the central pit and facets. 
horizontal and vertical axes to preserve the cross-sectional shape. If we move to the edge of the crystal, we can again find more shadowed regions with the right-angled islands and these appear to have a similar facet structure.

The ex situ microscopy was informative in that it suggests that exposure to air did not alter the basic facet structure that was apparent in the LEED after growth. However, there are key questions remaining: What is the structure and composition of the terrace? Are the islands formed in UHV indistinguishable from those imaged in air? How are the islands formed? To answer these questions, we undertook detailed spectroscopic imaging experiments of the x-ray absorption spectroscopy (XAS) by scanning the photon energies across the $\mathrm{Cr} L$ and $\mathrm{O}$ $K$ edges in total electron yield mode. In addition, scanning tunneling microscopy was employed to probe the smaller islands formed on the thinnest films.

\section{X-ray photoelectron emission microscopy}

Figure 4 shows an x-ray absorption spectra built up from a sequence of images taken as the photon energy is scanned. Islands were grown in situ (12-ML-thick film deposited at $803 \mathrm{~K}$ and annealed in UHV at the same temperature for 15 min) and show fine structure comparable to the ex situ FESEM results. Two XAS spectra are obtained by integrating the signal arising solely from the islands and separately the signal arising from the terrace in-between. These are then averaged to the number of pixels taken in each case. The drift correction (the sample is hot and thermal expansion moves the sample relative to the grazing incidence beam) in general means that the number of pixels analyzed varies from one frame to another so that the resulting XAS data are presented as the average intensity per pixel normalized to one at the preedge region. Sloping backgrounds and a significant fraction of the noise are due to this positional stability of the $(10 \times 10 \mu \mathrm{m})$ synchrotron beam, which tends to wander partially into and out of frame and can only be corrected for manually. Image contrast is due to the differing thickness and composition of the substrate and islands. Below the $\mathrm{Cr}$ absorption edge, a constant signal arises from the substrate absorption which is attenuated more strongly by the $\mathrm{Cr}$ islands so that they appear dark. At the $\mathrm{Cr}$ edge, the islands now photoemit more strongly than the substrate and the contrast is reversed with islands appearing bright. The XAS results for the area between islands also indicate that there is a spectroscopic contribution from a $\mathrm{Cr}$ wetting layer on the substrate between islands. The full sequence of images may be viewed in Supplemental Material. $^{28}$

\section{Scanning tunneling microscopy}

To further probe the structure of the wetting layer between the islands and the geometric structure of the islands, STM experiments were performed on both substrate crystals in the low $\mathrm{Cr}$ coverage regime from clean to $\sim 5 \mathrm{ML}$. Figure 5(a) shows small island arrays dewet from the highly stepped surface after the deposition of $\sim 3 \mathrm{ML} \mathrm{Cr}$, which generates the LEED pattern shown in Fig. 1(d). The islands are uniformly $\sim 2 \mathrm{~nm}$ high [Fig. 5(b)] and many have already formed a central pit or depression central region of the upper facet. The larger islands (with a length of $\sim 140 \mathrm{~nm}$ ) have very similar widths of the adjoining facets to the width of the smaller islands. This characteristic island or facet width is $51 \pm 1 \mathrm{~nm}$ with a standard deviation of $8 \mathrm{~nm}$. However, the islands do not seem to display a characteristic anisotropic shape, which is what could be expected to arise from a growth regime following the Tersoff and Tromp ${ }^{13}$ model in which the short axis of the islands is epitaxial and strained, but the long axis of the island is relaxed to the bulk lattice constants.

Between the islands, the XPEEM indicates a layer of chromium is present. The STM images of this region display the characteristic step edges of the W(100) substrate overlayed with a fine network of structure with a predominant fourfold symmetry [Fig. 5(c)]. The step edges are more structured in the Cr-covered substrate than on a clean sample. This area of substrate is a large fraction of the total surface area

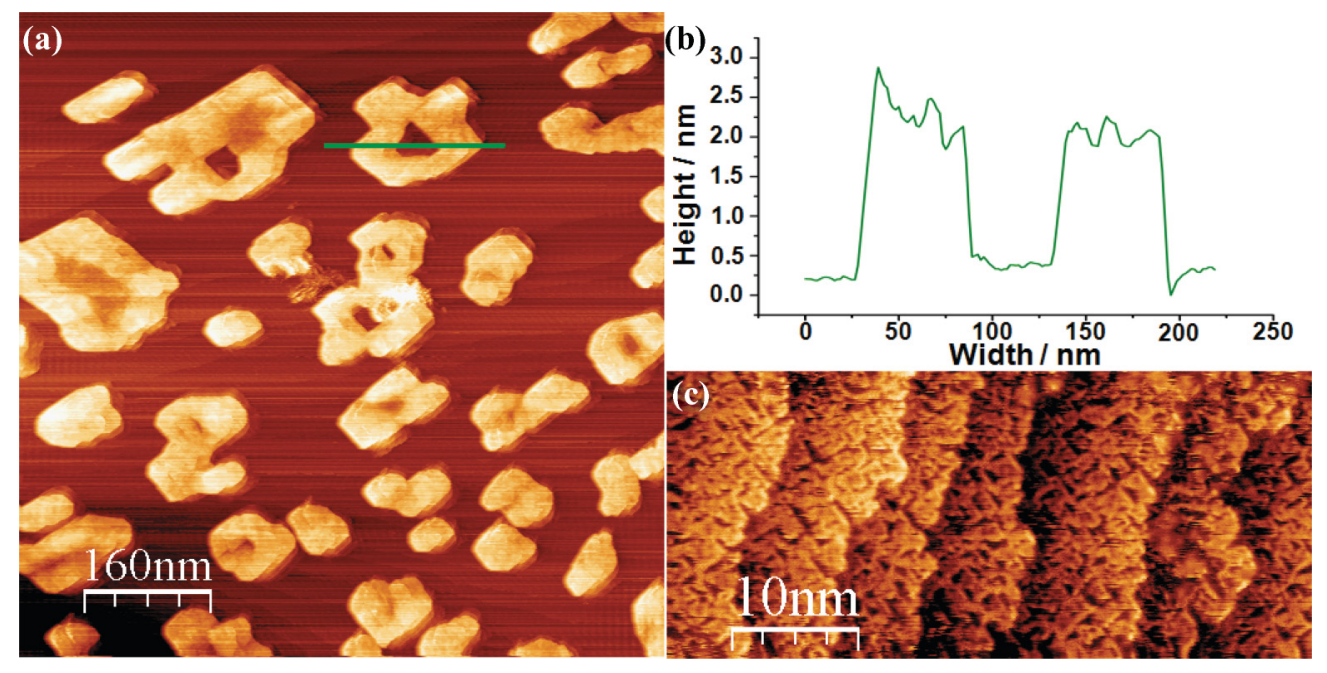

FIG. 5. (Color online) (a) $(8000 \times 8000 \AA$, $1 \mathrm{nA} 1 \mathrm{~V})$ STM image of Cr islands formed on W(100) surface after 3-ML deposition; (b) a line profile across a typical island showing steep sides, tilted upper terrace, and central pit; (c) $(500 \times 240 \AA ⿻$, $0.5 \mathrm{nA} 0.5 \mathrm{~V}) \mathrm{STM}$ image of the substrate imaged between dewetted islands showing distinctive $\mathrm{Cr}$ morphology decorating the substrate terraces. 
and we therefore expect it to appear strongly in the LEED patterns. Indeed, the W(100) integral order spots are sharp and intense and so we believe that this structured layer is in fact the pseudomorphic Cr layer. Despite only a small sample of images in STM, we did not find any multilayer islands on top of the terraces so we suggest that the $\mathrm{Cr}$ wetting layer is $\sim 1 \mathrm{ML}$ thick. However, the fine patterning of this layer which is apparent and generates a blocky motif suggests that there may be a noninteger coverage of $\mathrm{Cr}$ such that the pseudomorphic $\mathrm{Cr}$ is not a complete monolayer. Similar motifs have been shown to form for annealed submonolayer $\mathrm{Cr}$ on W(110) (Ref. 30) with the formation of high-aspect-ratio vacancy nanolines.

For higher coverages, fully facetted islands may be formed by annealing as shown in Fig. 6. This island fills the full frame of our widest STM scan [Fig. 6(a), $800 \mathrm{~nm}$ ] and was grown on the crystal with the lower step density. The facets of this nanocrystal are well defined as can be seen in the accompanying line profile [Fig. 6(b)] across the central pit. The main facets appear to be oriented between $4.8^{\circ}$ and $7^{\circ}$ from horizontal, with normals pointing into the center of the island, with steeper facets on the inside and outer edges at $\sim 24^{\circ}$. The substrate steps can be imaged in the lower right corner of the image and allow us to properly define the substrate plane and the local average terrace width of $\sim 80 \AA$.

\section{E. UV photoelectron emission microscopy}

Having established chemical composition and geometry of these structures, the most prominent open question remains as to how they form. To shed light on this, we employ the time-resolved in situ imaging capability of PEEM to image the dewetting of structures from metastable multilayer films. In the selected sequence of images shown in Fig. 7, the sample is maintained at $842 \mathrm{~K}$ for Figs. 7(a)-7(d) to trigger the dewetting and then reduced to $826 \mathrm{~K}$ to follow growth. The full sequence of images may be viewed in Supplemental Material. ${ }^{28}$ The compact islands nucleate rapidly and appear as small dots with a bright penumbra that grows out from each center. The image contrast in UV PEEM is derived from the intensity of secondary electrons emitted from the sample under $\sim 5 \mathrm{eV} \mathrm{Hg}$ lamp illumination and is dominated by work-function contrast between differing materials and/or structure and especially roughness. The bright region is caused by $\mathrm{Cr}$ diffusion to the islands leaving a thinner and rougher area with lower work function. Over time, the islands grow [Figs. 7(e)-7(h)] by denuding the $\mathrm{Cr}$ from the film, and as they get taller they begin to throw shadows (the UV light is at a grazing angle of incidence). Finally, a low density of compact, mostly square but some $L$ shaped islands are formed on a uniform background. Analysis of the rate at which the denuded region grows around each island shows that the radial distance of the rim scales as approximately $t^{1 / 2}$. The spontaneous dewetting was observed to occur over a range of film thicknesses on the highly stepped crystal (experiments were not performed on the lower step density substrate). If deposition onto the clean surface was undertaken at elevated temperatures $(\sim 673 \mathrm{~K})$, the island formation was identical on both crystals, and predominantly square hollow centered facetted islands result.
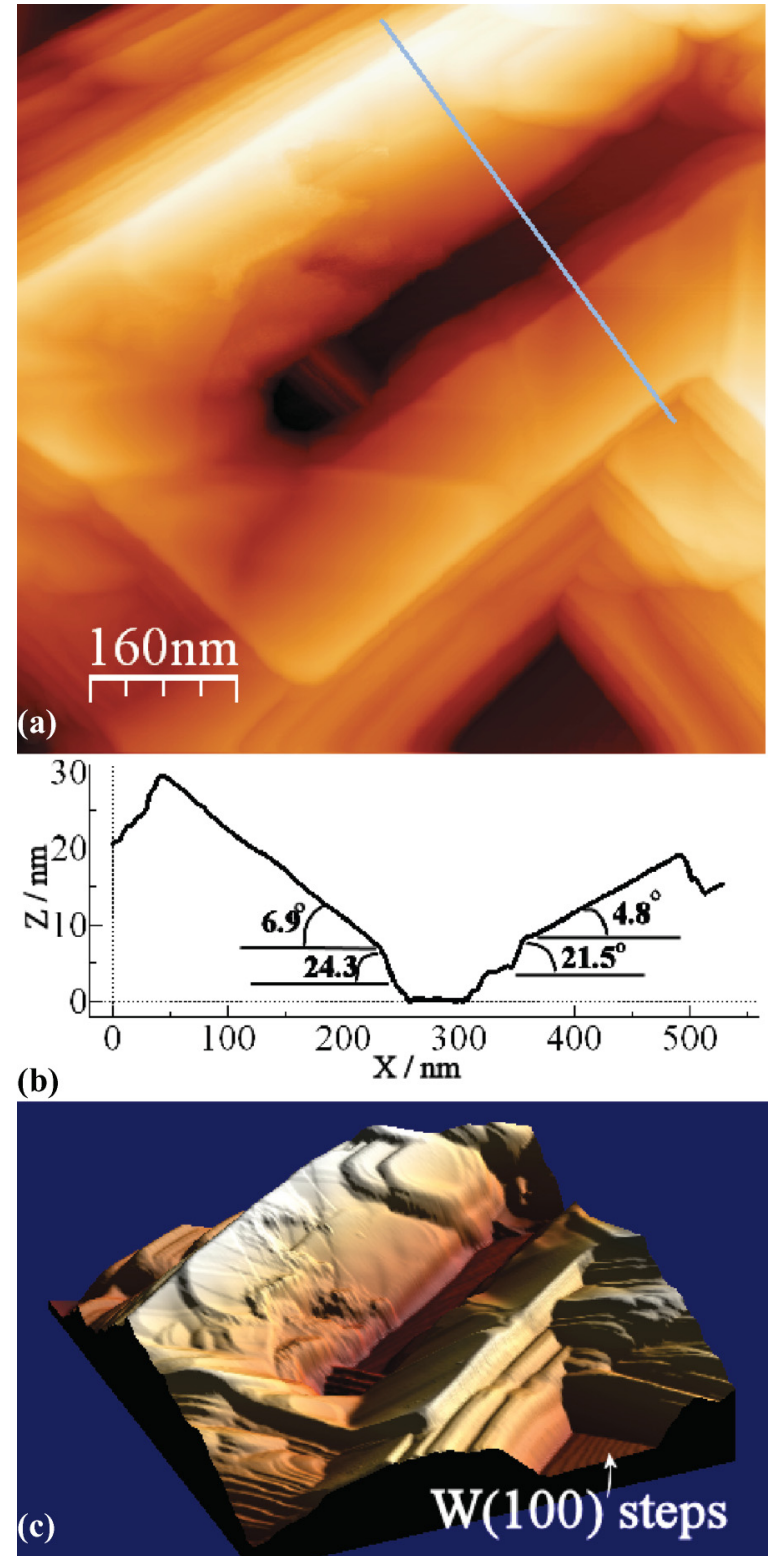

FIG. 6. (Color online) (a) STM image $(8000 \times 8000 \AA$, $0.01 \mathrm{nA} 0.5 \mathrm{~V}$ ) of a large facetted island grown on W(100) with line profile highlighted; (b) line profile across the dominant facet structure showing steep and shallow facets; and (c) three-dimensional rendering of (a) showing morphology and the observation of substrate steps with the local structure of $\sim 80$ - $\AA$ terrace widths.

\section{DISCUSSION}

The lattice mismatch between $\mathrm{Cr}$ overlayers and $\mathrm{W}$ substrate is the driving force in dewetting of the film due to an increase in strain with film thickness. Relief from the strain is commonly seen through the formation of dislocations in overlayers where an extra layer of atoms can be inserted (or removed) periodically to match a sublattice or through islanding where overlayers adopt free surfaces that can relax to the bulk lattice constant. The transformation from strained film to nanostructured overlayer is usually controlled by the kinetics. Recently, much work has focused on dewetting from stepped substrates and island drift in the down-step 


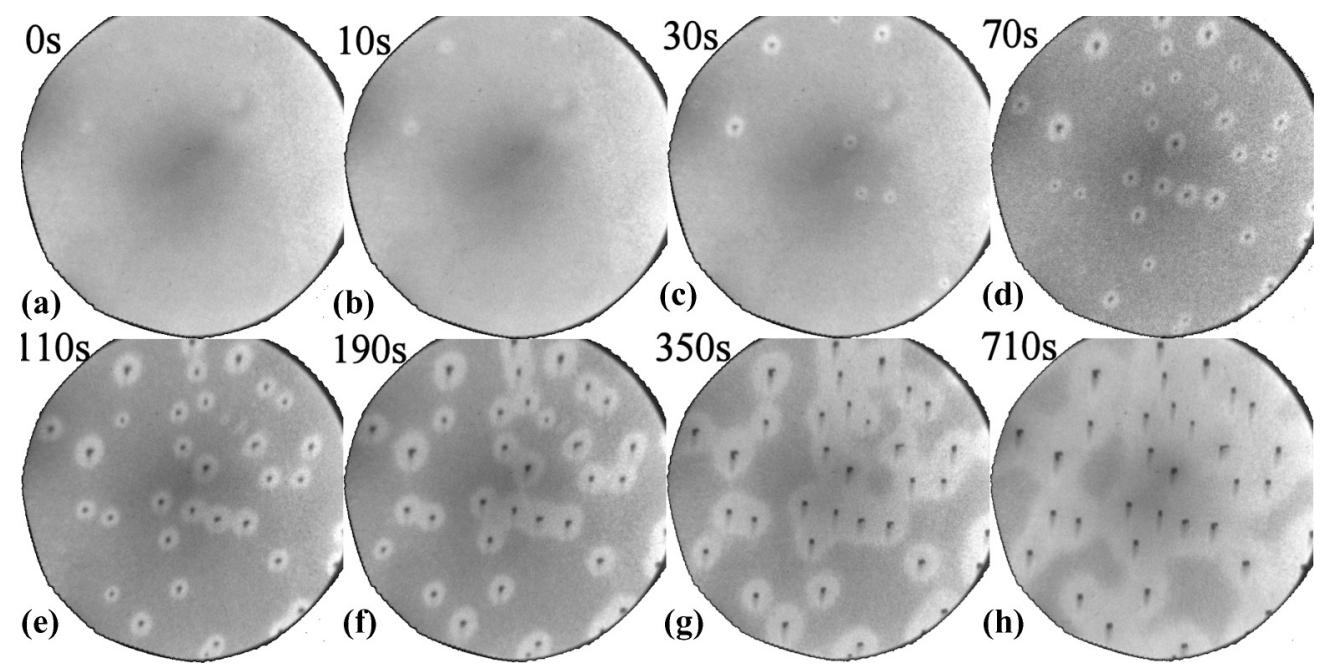

FIG. 7. Time-lapse UV-PEEM images (40- $\mu \mathrm{m}$ field of view) showing the dewetting of chromium multilayers from W(100). Images (a)-(f) were acquired as the sample temperature was slowly raised to $\sim 842 \mathrm{~K}$ to initiate nucleation. The temperature was reduced to $\sim 826 \mathrm{~K}$ to follow growth in (e)-(h). Time zero in image (a) is the first frame with any sign of nucleation.

direction. In the latter case, thicker films are energetically much more favorable than thinner films and so step edges nucleate dewetting by aggregation of material at the down step at the cost of thinner layers at the upper terrace. Larger islands on substrates with high step density follow a similar trend with islands adopting a flat-topped mesa structure, which have been seen to migrate in the down-step direction as material preferentially thickens the thicker end of the island.

In our work, we find that the highly stepped crystal can nucleate islands from dewetting of a metastable film, as described above. Additionally, spontaneously nucleated islands can also be readily grown by $\mathrm{Cr}$ deposition at elevated temperature onto a W(100) substrate with either a high or relatively low step density. In all scenarios, elevated temperature deposition or post deposition anneal on either highly stepped or less stepped substrates, and the same island morphology arises. The islands are aligned with the substrate, and do not seem to be particularly influenced by the local step orientation. This suggests that the resulting island shape is particularly energetically stable and not simply driven by growth kinetics, which would differ markedly for the four combinations of methods and substrates considered. In the comparable $\mathrm{Fe}$ on $\mathrm{W}(100)$ system with almost identical lattice mismatch, compact islands dominate for low- and high-temperature growth, while at intermediate temperatures, flat stripes of 4-ML-thick $\mathrm{Fe}$ form with clear dislocation lines. ${ }^{9}$ The difference in behavior may be due to the higher stiffness of Cr leading to higher strain energies for a given lattice mismatch and a more brittle character.

The measured tilts of the facets on the islands with respect to the substrate terraces are between $4.8^{\circ}$ and $7^{\circ}$ for the upper terraces and $\sim 18^{\circ}-25^{\circ}$ for the steeper sides (both inner and outer). The LEED indicates that the dominant top facets are $\operatorname{Cr}(100)$ surfaces tilted by $5^{\circ}-7^{\circ}$ from the $\mathrm{W}(100)$ substrate. If 11 or $12 \mathrm{Cr}$ unit cells ( 31.68 or $34.56 \AA$ ) grow on 10 or $11 \mathrm{~W}$ substrate cells ( 31.6 or $34.76 \AA$ ), then the total mismatch is minimal. Restricting the lattice to a cubic symmetry suggests a tilt of $4^{\circ}$ or $5.8^{\circ}$, which would allow integer numbers of cells to remain in registry. The steeper side walls make an angle of $\sim 160^{\circ}$ to the upper inclined (100) facets. Such an angle could be naturally accomplished by terminating the tilted bulk bcc $\mathrm{Cr}$ structure in a (210) facet, which appears at $153^{\circ}$ to the (100). Interestingly, McCarty et al. showed that the formation of nanostripes elongated along the W[001] by dewetting of $\mathrm{Cr}$ from W(110) resulted in (120)-orientated side facets. ${ }^{10}$ Therefore, the cross-sectional shape of the $\mathrm{Cr}$ islands appears to be of a bulk Cr lattice bounded by $\{210\}$ planes on the side and with $\{100\}$ upper facets. This structure is rotated in the surface plane by $5^{\circ}-7^{\circ}$ degrees towards the center of the island and repeated for each equivalent $\{010\}$ direction in plane to form the square-based walled islands. A schematic of the cross-section epitaxial and facet structure of the $\mathrm{Cr}$ on $\mathrm{W}$ is shown in Fig. 8.

The dynamics of the growth morphology is not yet clear although our microscopy at key growth stages has identified a low coverage, untilted, thin square dewetted island at early stages of dewetting, rapid mass transport to these islands as the film dewets, and a final island habit with well-defined facets. The wetting layer has been identified spectroscopically and shown to be pseudomorphic by LEED and nanostructured

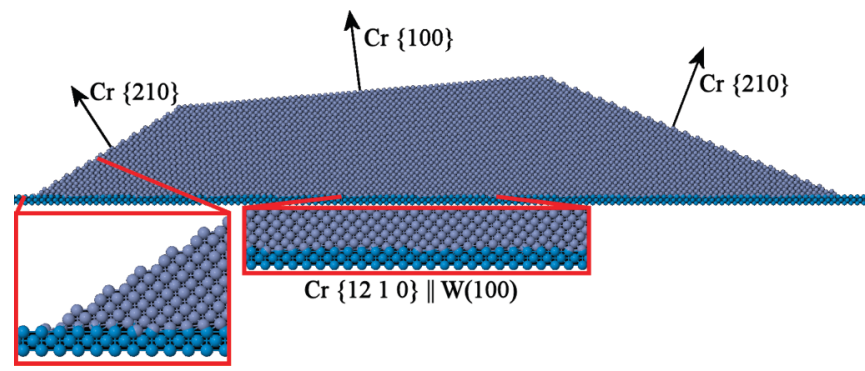

FIG. 8. (Color online) Structural model of the cross section through the wall of a Crisland on W(100). The atoms have been shown overlapping at the $\mathrm{Cr}-\mathrm{W}$ interface to clarify the epitaxial arrangement. The island center is at the left-hand edge and island outer edge on the right. 
STM imaging. The intensity of the pseudomorphic Cr LEED spots taken at the same energy but at coverages of two or more ML vary significantly from both the clean W(100) and from a 1-ML Cr film. While we are currently investigating this in detail, and will report in the future, ${ }^{27}$ the LEED-IV suggest that the wetting layer (present above 2-ML depositions where islands have formed) is not identical to the pseudomorphic monolayer. The STM confirms that the wetting layer is incomplete and hence may be just under 1 or 2 ML thick.

Of the various theoretical models that are used to describe the equilibrium shapes of islands, few appear to predict many of the key features and especially the facetted closed-ring structures shown here. The Wulf-Kaishew theorem presented in detail by Müller and Kern, ${ }^{31}$ in which the equilibrium shape (ES) of strained crystals can be predicted, showed that such shapes were compact without hollow centers and were symmetrical but could change shape as they grew due to the introduction of dislocations in the strained bulk. With each dislocation event, the ES of the truncated pyramids flattened considerably as the effective mismatch was reduced, and the upper facet became enlarged. There was no discussion of crystal reorientation to reduce stress as is seen with each of the sidewalls of our islands. The work of Tersoff and Tromp ${ }^{13}$ in describing elongation of trapezoidal cross section supported crystals beyond a characteristic size perhaps best describes the basis of the formation of our sidewalls, especially when considering the very long-sided $L$-shaped islands. However, this theory does not predict the closed-ring structures that we find, nor the tilting of the sidewalls. The tilting and creation of asymmetry in shapes can be introduced by consideration of epitaxial islands on vicinal surfaces ${ }^{15}$ in which the ES of semiconductor quantum dots develops from halfpyramid through pyramid, half-dome, dome, half-barn, and barn shapes with increasing volume for notional $1^{\circ}-4^{\circ}$ miscuts. Each structure has more facets in its ES than its predecessor. For smaller miscut angles, the half-dome and half-barn do not appear in the phase diagram, while for larger than $4^{\circ}$, only the half structures are predicted. At zero miscut, the structures are predicted to be symmetric. The pyramid structure formed for small miscuts most closely aligns with the cross-sectional shape of our island sidewalls (albeit ours are metal while the model is developed for semiconductors). However, our substrates are not particularly vicinal (the measured step density of our two crystals correspond to $1.3^{\circ}$ and $0.4^{\circ}$ miscuts) and the morphology of our islands is square and therefore fourfold symmetric rather than having one preferred direction induced by the substrate. Islands grown on vicinal surfaces develop flat-topped mesa structures that migrate down the substrate step direction when growing to generate thicker structures with lower overall strain energy per atom (thin films have a strained bulk driven by the free surfaces, while thicker films have strained surfaces and relatively relaxed bulks). ${ }^{10}$ A similar mechanism may hold in our system as tilting the crystallite maintains the bulk lattice constants and allows a low-index well-defined top facet to form. This generates a $\mathrm{Cr}\left\{\begin{array}{lll}12 & 1 & 0\end{array}\right\}$ interface parallel to the substrate W(100). The $\mathrm{Cr}\left\{\begin{array}{lll}12 & 1 & 0\end{array}\right\}$ interface then takes on the role of the vicinal substrate appearing as a $5^{\circ}$ miscut to the $\operatorname{Cr}(100)$. Thus, the same basic physics can hold and the crystallite grows or moves to thicken the thick edge at the expense of the thin. There are four equivalent $\operatorname{Cr}\left\{\begin{array}{lll}12 & 1 & 0\end{array}\right\}$ planes that are parallel to $\mathrm{W}(100)$, so we can naturally describe all four sidewalls without recourse to a vicinal substrate.

The final point that needs discussion is the predominance of closed-ring structures. With four equivalent sidewalls, one might expect zigzag morphologies or long meandering chainlike nanostructures to form. Instead, only closed squares or $L$ shapes form and these always have a thicker outer edge than inner. Thus, we suggest that there is an energetic preference to forming right-angle junctions between two sidewalls in which the tilts of the sidewalls point inward. These junctions lie on $\{110\}$ planes which are slip planes in bcc materials and, hence, are the locations where misfit dislocations and extra planes can be preferentially inserted, as shown by TEM in epitaxial Cr films. ${ }^{32}$ The stability of ring structures then arises due to the elimination of free facets at the end of the sidewalls. In this geometry, any sidewall motion (as described above for vicinals) will be outward from the center of the island and be constrained by the necessity to keep the structure closed. Growing islands would then tend to extend outward rather than collapse to form compact islands.

In conclusion, we report the surprising formation of squarebased facetted islands with linear dimension of the order of $500 \mathrm{~nm}$ upon dewetting of a $\mathrm{Cr}$ multilayer on W(100). We show that these square islands are composed of inclined facets surrounding a depressed center such that the facet slopes inward with the outer edges of the islands thicker than the centers. In situ UV and x-ray photoelectron emission microscopy allied to spatially resolved spectroscopy throws considerable light on the nature of the dewetting and shows that the metal surface between the islands remains covered by a thin pseudomorphic wetting layer of just less than 1(or possibly 2-) ML thick. Low-energy electron diffraction and scanning tunneling and atomic force microscopies allow quantification of facet slopes and we identify a predominance of $\operatorname{Cr}(100)$ facets tilted by $\pm 5^{\circ}$ with respect to the substrate normal bounded by (210) planes at $\sim 24^{\circ}$ with respect to the substrate. The $\mathrm{Cr}$ islands adopt the bulk $\mathrm{Cr}$ lattice constant and are oriented with each sidewall crystallite having a plane of $\mathrm{Cr}\{1210\}$ parallel to the substrate W(100). We believe that the morphologies identified here by various techniques represent a class of shapes dictated by the crystallite reorientation, and that the pseudovicinal interface may further drive the island dynamics and influence mobility and growth. It remains a challenge to theory to fully understand their stabilization.

\section{ACKNOWLEDGMENTS}

We acknowledge the Science and Technology Facilities Council for provision of experimental beamtime at Diamond under award SI75 and the Royal Society/Wolfson foundation for provision of a laboratory refurbishment grant. We thank H. Marchetto for the provision of XPEEM data analysis software, in particular the drift correction of the images, and P. Harris of the Centre for Advanced Microscopy for taking the ESEM images. We also acknowledge the excellent support staff at the Diamond synchrotron that set up and enabled science on this new facility and similarly V. Dhanak and colleagues at the former Daresbury synchrotron. 
*R.A.Bennett@Reading.ac.uk

${ }^{1}$ A. Kubetzka, M. Bode, O. Pietzsch, and R. Wiesendanger, Phys. Rev. Lett. 88, 057201 (2002).

${ }^{2}$ M. Ziegler, N. Ruppelt, N. Néel, J. Kröger, and R. Berndt, Appl. Phys. Lett. 96, 132505 (2010).

${ }^{3}$ G. Rodary, J.-C. Girard, L. Largeau, C. David, and O. Mauguin, Appl. Phys. Lett. 98, 082505 (2011).

${ }^{4}$ X. Wei, P. Jiang, and G. J. Che, Chin. Phys. Lett. 22, 1232 (2005).

${ }^{5}$ L. Zhang, M. Kuhn, U. Diebold, and J. A. Rodriguez, J. Phys. Chem. B 101, 4588 (1997).

${ }^{6}$ P. J. Berlowitz and N. D. Shinn, Surf. Sci. 209, 345 (1989).

${ }^{7}$ D. Guo, Q. Guo, M. S. Altman, and E. G. Wang, J. Phys. Chem. B 109, 20968 (2005).

${ }^{8}$ K. L. Man, Q. Guo, and M. S. Altman, Surf. Sci. 600, 1060 (2006). ${ }^{9}$ W. Wulfhekel, F. Zavaliche, R. Hertel, S. Bodea, G. Steierl, G. Liu, J. Kirschner, and H. P. Oepen, Phys. Rev. B 68, 144416 (2003).

${ }^{10}$ K. F. McCarty, J. C. Hamilton, Y. Sato, A. Saá, R. Stumpf, J. de la Figuera, K. Thürmer, F. Jones, A. K. Schmid, A. A. Talin, and N. C. Bartelt, New J. Phys. 11, 043001 (2009).

${ }^{11}$ I. V. Shvets, S. Murphy, and V. Kalinin, Surf. Sci. 601, 3169 (2007).

${ }^{12}$ A. Cazacu, S. Murphy, and I. V. Shvets, Phys. Rev. B 73, 045413 (2006).

${ }^{13}$ J. Tersoff and R. M. Tromp, Phys. Rev. Lett. 70, 2782 (1993).

${ }^{14}$ M. S. J. Marshall and M. R. Castell, Phys. Rev. Lett. 102, 146102 (2009).

${ }^{15}$ B. J. Spencer and J. Tersoff, Appl. Phys. Lett. 96, 073114 (2010).

${ }^{16}$ M. Dufay and O. Pierre-Louis, Phys. Rev. B 81, 041407 (2010).

${ }^{17}$ K. F. McCarty, Nano Lett. 6, 858 (2006).

${ }^{18} \mathrm{We}$ note that the growth of quantum ring-shaped structures has been reported for lattice mismatched semiconductor systems using droplet epitaxy in which surface supported Ga droplets are crystallized into GaAs by starting and stopping the As flux. This is a fundamentally different process to that reported here;
T. Mano, T. Kuroda, S. Sanguinetti, T. Ochiai, T. Tateno, J. Kim, T. Noda, M. Kawabe, K. Sakoda, G. Kido, and N. Koguchi, Nano Lett. 5, 425 (2005).

${ }^{19}$ R. A. Bennett, J. S. Mulley, M. A. Newton, and M. Surman, J. Chem. Phys. 127, 084707 (2007).

${ }^{20}$ N. D. McCavish and R. A. Bennett, Surf. Sci. 546, 47 (2003).

${ }^{21}$ R. E. Palmer and G. Lloyd (unpublished).

${ }^{22}$ P. Stone, S. Poulston, R. A. Bennett, N. J. Price, and M. Bowker, Surf. Sci. 418, 71 (1998).

${ }^{23}$ H. A. Etman, Z. V. Zheleva, G. Held, and R. A. Bennett, J. Phys. Chem. C 115, 4191 (2011).

${ }^{24}$ I. K. Robinson, A. A. MacDowell, M. S. Altman, P. J. Estrup, K. Evans-Lutterodt, J. D. Brock, and R. J. Birgeneau, Phys. Rev. Lett. 62, 1294 (1989).

${ }^{25}$ http://www.diamond.ac.uk/Beamlines/Beamlineplan/I06/ TechSpecs.htm

${ }^{26}$ I. Horcas, R. Fernández, J. M. Gómez-Rodríguez, and J. Colchero, Rev. Sci. Instrum. 78, 013705 (2007).

${ }^{27}$ R. A. Bennett, J. S. Mulley, H. A. Etman, and V. R. Dhanak (unpublished).

${ }^{28}$ See Supplemental Material at http://link.aps.org/supplemental/ 10.1103/PhysRevB.86.045454 for Fig. SM1 of selected ESEM images of surface feature directed nonuniform $\mathrm{Cr}$ structures, a movie SM1 taken from the X-PEEM XAS measurements, and a movie SM2 of the dewetting of a metastable film in UV-PEEM.

${ }^{29}$ VEECO MSCT- Microlever Probes contact mode SiN tips with square based pyramidal 35 degree sides and a $10 \mathrm{~nm}$ nominal tip radius: https://www.veecoprobes.com

${ }^{30}$ E.-P. Kim, T.-H. Kim, S.-T. Kim, Y. Kuk, and S.-J. Kahng, Thin Solid Films 441, 317 (2003).

${ }^{31}$ P. Müller and R. Kern, Surf. Sci. 457, 229 (2000).

${ }^{32}$ C.-M. Wang, T. C. Kaspar, V. Shutthanandan, A. G. Joly, and R. J. Kurtz, Acta Mater. 59, 4274 (2011). 